\title{
A Rare Case of Pre-Existing Deep Venous Thrombosis in the Peroneal Vein of a Free Osteocutaneous Fibula Flap
}

\author{
Satoshi Hayashi, MD ${ }^{1}$ Kenji Kawamura, MD $\quad$ Naoki Maegawa, MD ${ }^{1} \quad$ Akito Nakanishi, MD ${ }^{1}$ \\ Yasushi Mizutani, MD ${ }^{1}$ Akinori Okuda, MD ${ }^{1}$ Shinpei Kurata, MD ${ }^{1}$ Takamasa Shimizu, MD ${ }^{1}$ \\ Tsutomu Kira, MD ${ }^{1}$ Shohei Omokawa, MD ${ }^{1}$ Yasuhito Tanaka, MD ${ }^{1}$
}

${ }^{1}$ Department of Orthopaedic Surgery, Nara Medical University, Nara, Japan

Address for correspondence Kenji Kawamura, MD, Department of Orthopaedic Surgery, Nara Medical University, 840 Shijyo-cho,

J Reconstr Microsurg Open 2018;3:e74-e77.

Kashihara, Nara 634-8522, Japan (e-mail: kkenji@naramed-u.ac.jp).

\begin{abstract}
Background Deep venous thrombosis (DVT) of the lower leg is a widespread medical problem and is associated with hypercoagulable states. Although spontaneous thrombosis of the peroneal vein of a fibula flap is theoretically possible, few cases of asymptomatic DVT of the peroneal vein encountered during fibula flap harvest have been reported.

Case Report A 45-year-old man presented with segmental bone defects of the tibia resulting from a fall. Treatment with a free vascularized osteocutaneous fibula flap from the contralateral lower leg was scheduled. Intraoperatively, diffuse thrombi were found in the peroneal venous system. After removal of thrombi, the fibula was grafted with anastomosis of the peroneal vessels to the recipient posterior tibial vessels. The skin paddle of the flap became congested after surgery; exsanguination of the skin paddle was performed with multiple skin incisions to relieve congestion. Despite treatment, the skin paddle became partially necrotic and additional skin grafting was performed.

Keywords

- osteocutaneous fibula flap

- deep venous thrombosis

- peroneal vein

Bone union of the grafted fibula was achieved 5 months after initial surgery.

Conclusions To the best of our knowledge, only six cases of pre-existing DVT in the peroneal vein of a free osteocutaneous fibula flap have been reported. In three of these cases, the fibula flap was successfully grafted. Although successful free osteocutaneous fibula flap surgery might be possible in patients with peroneal venous thrombosis, careful preoperative evaluation is recommended for high-risk patients.
\end{abstract}

Vascularized free osteocutaneous fibula flaps have become widely used for reconstruction of bone and soft tissue defects of the lower leg since the first description of the technique by Taylor et al in $1975 .^{1}$ Successful harvesting and grafting of a fibula flap depends on many factors, including adequate vascular inflow and outflow systems. Although preoperative evaluation of the arterial system of the lower leg is commonly performed with several modalities, including angiography, computed tomography (CT) angiography, magnetic resonance arteriography, and Doppler's ultrasonography, the venous

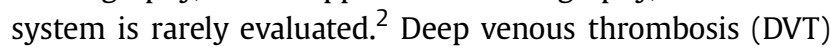
of the lower leg is a widespread medical problem associated with hypercoagulable states, including malignancy, vessel wall injury, and prolonged periods of immobilization. ${ }^{3}$ Sponta-

received

June 28, 2018

accepted after revision

August 30, 2018
DOI https://doi.org/

$10.1055 / \mathrm{s}-0038-1675410$ ISSN $2377-0813$ neous thrombosis of the peroneal vein of a fibula flap is theoretically possible; however, to the best of our knowledge, only six cases of asymptomatic DVT of the peroneal vein encountered during fibula flap harvest have been reported. ${ }^{4-8}$ We present a rare case of pre-existing DVT in the peroneal vein of a free osteocutaneous fibula flap for reconstruction of segmental bone defects of the tibia, and we discuss possible salvage procedures in this situation.

\section{Case Report}

A 45-year-old man sustained right femoral shaft fracture, right tibial open fracture, and bilateral patella fractures in a fall. The right femoral shaft fracture and bilateral patella

Copyright $\odot 2018$ by Thieme Medical Publishers, Inc., 333 Seventh Avenue, New York, NY 10001, USA. Tel: +1(212) 584-4662.
License terms

()(1) $\odot \circledast$ 
fractures were internally fixed at another hospital. The right tibial open fracture was temporarily stabilized with an external fixator after debridement. Fourteen days after the injury, the patient was referred to our hospital for reconstruction of the right lower extremity. He presented with segmental bone defects of the distal right tibia ( - Fig. 1). Repair with a free vascularized osteocutaneous fibula flap from the contralateral lower leg was scheduled. Preoperative CT angiography of the lower legs revealed no vascular anomalies in either leg. The vascularized fibula flap was harvested under the tourniquet control. After harvesting of the fibula flap from the left leg, diffuse thrombi were found in the peroneal venous system (-Fig. 2). Intraluminal thrombi in the peroneal vein were removed by picking with forceps, and the lumen of the peroneal vein was washed out with heparin-added physiological saline solution. After removal of thrombi, the fibula was fixed at the recipient site and peroneal vessels were anastomosed to the recipient posterior tibial vessels. The external fixator was reapplied to provide stability. The heparin sodium $(15,000$ units per day) was administered intravenously for a week after surgery. Then, rivaroxaban was administered orally for 3 weeks to prevent pulmonary embolism. The skin paddle of the flap became congested after surgery; exsanguination of the skin paddle with multiple skin incisions was performed to relieve congestion (-Fig. 3). Despite this treatment, the skin paddle became partially necrotic and additional skin grafting was performed (-Fig. 4). Bone union of the grafted fibula was achieved 5 months after the initial surgery (-Fig. 5). Angiography or bone scintigraphy was not performed after surgery

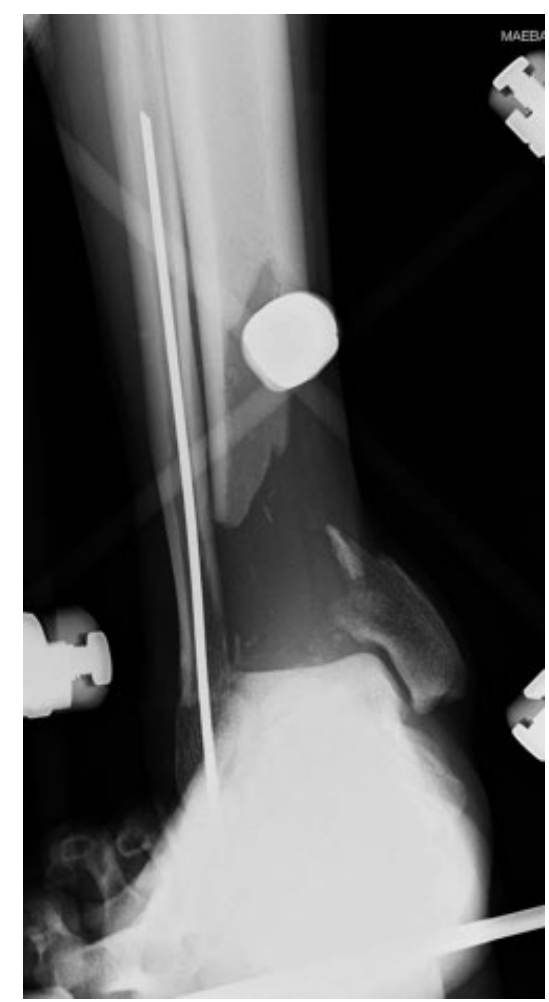

Fig. 1 Preoperative X-ray. Segmental bone defects are presented in the right distal tibia.

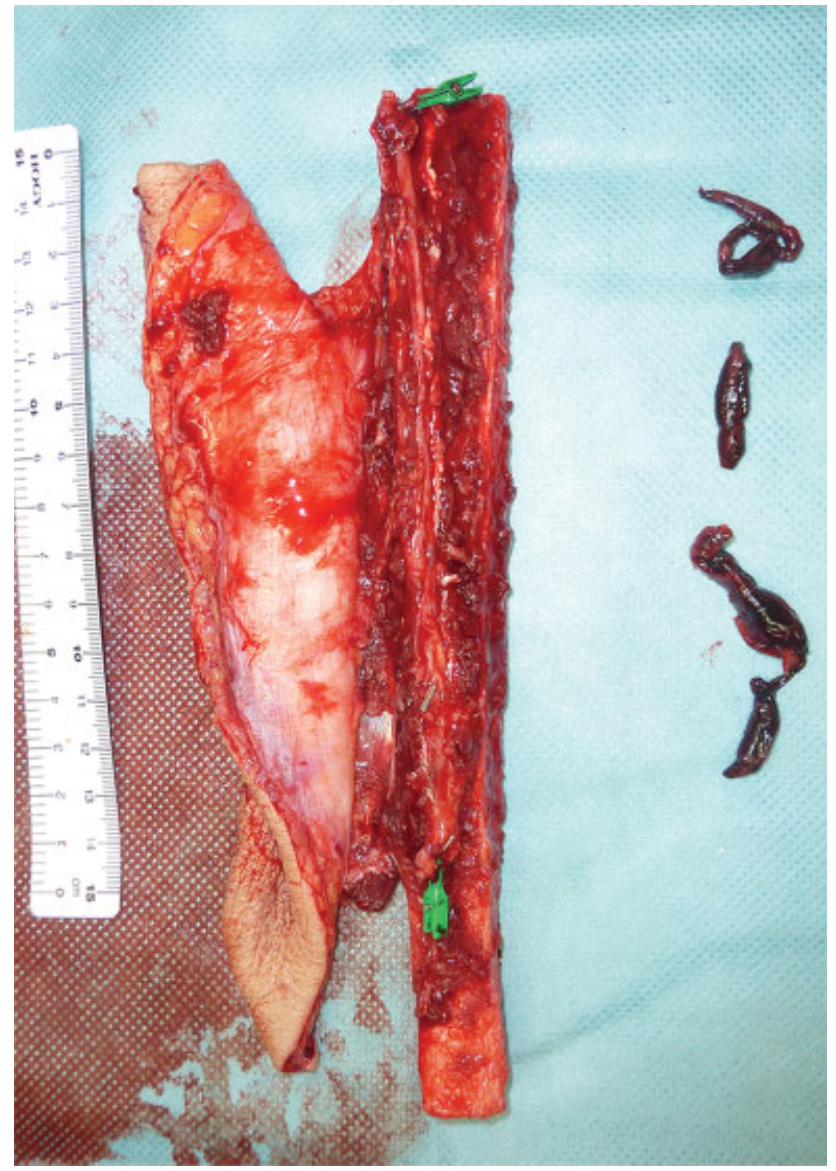

Fig. 2 Harvested fibula flap. Diffuse thrombi are present in peroneal venous system of fibula flap.

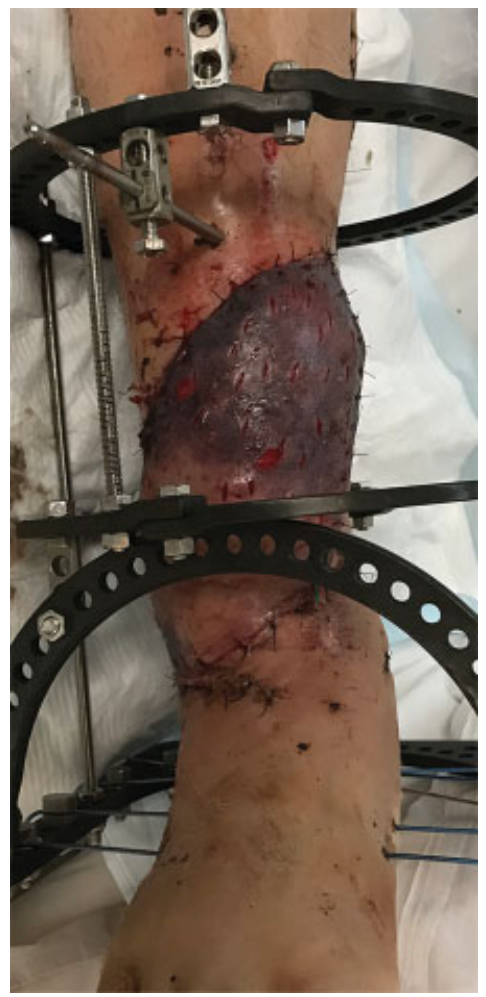

Fig. 3 Appearance of skin paddle at 3 days after surgery. Exsanguination of the skin paddle was performed with multiple skin incisions to relieve congestion after surgery. 


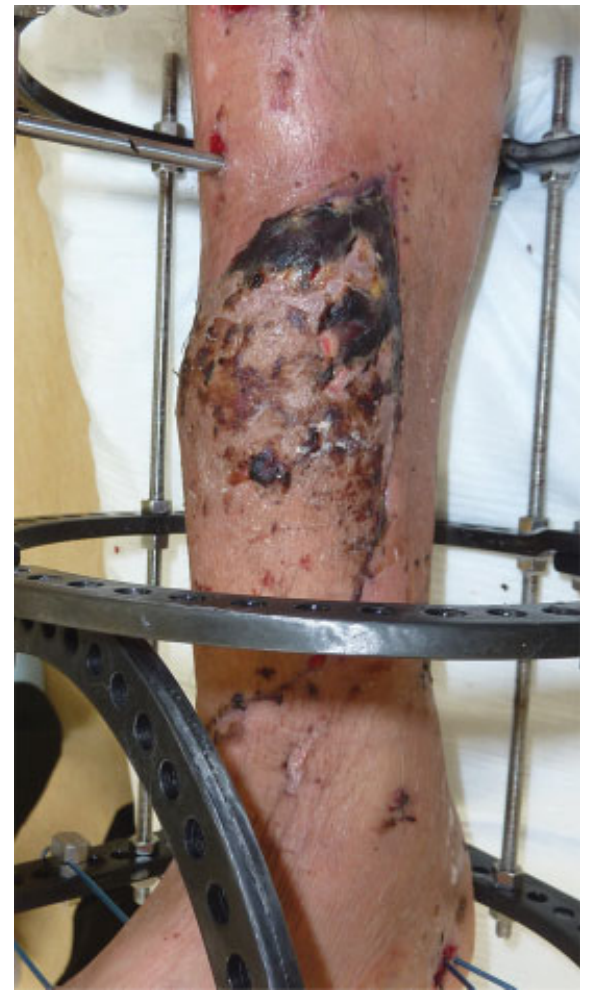

Fig. 4 Appearance of skin paddle at 3 weeks after surgery. The skin paddle became partially necrotic.
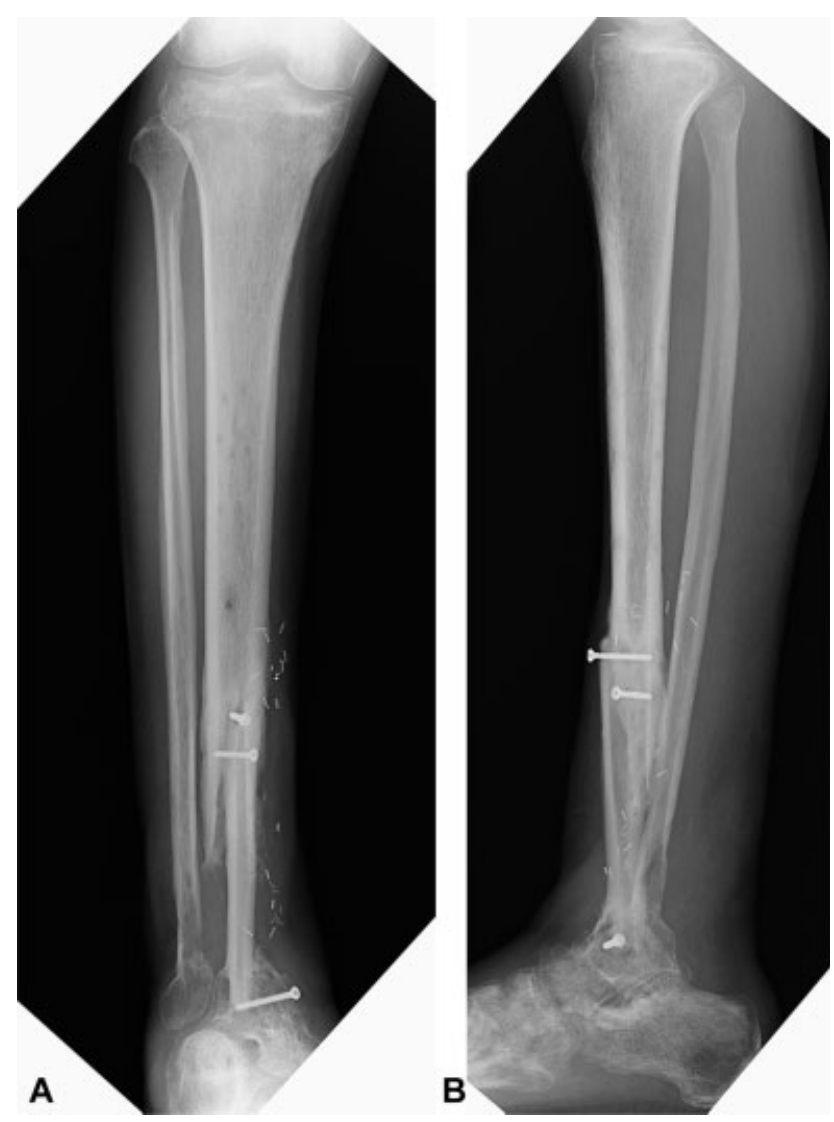

Fig. 5 Postoperative X-rays. Bone union of the grafted fibula was achieved 5 months after the initial surgery. (A) Anteroposterior and (B) lateral X-rays. to demonstrate the vascularization of the fibula flap. At the final follow-up 17 months after the surgery, he could walk without assistance.

\section{Discussion}

Deep venous thrombosis of the lower leg can occur in patients with prolonged periods of immobilization after trauma. ${ }^{3}$ Our patient had 20 days of bed rest before flap surgery. In addition, the patient's left leg, from which a free vascularized osteocutaneous fibula flap was harvested, had been immobilized in a splint after internal fixation of a patella fracture. The patient was classified in the highest risk level for developing DVT; according to the Caprini scoring system. ${ }^{9}$ Enhanced CT was performed preoperatively to evaluate the vascularity of the bilateral lower legs. Because the thrombi were not seen on the venous phase of preoperative enhanced CT scans, we excluded the possibility of DVT and did not perform lower limb venous ultrasound examination. However, we encountered thrombi in the peroneal venous system of the donor leg during fibula flap harvesting. We routinely investigate details of the arterial system in the lower legs before fibula flap surgery because the procedure is contraindicated if arterial disease is present. However, the venous system is rarely evaluated. The peroneal vein is a common site for DVT development. ${ }^{10}$ In patients at high risk of developing DVT, as in the present case, careful preoperative evaluation of the peroneal venous system by ultrasound should be performed.

There have been few descriptions of DVT during fibula flap harvest. To the best of our knowledge, only six cases of preexisting DVT in the peroneal vein of a free osteocutaneous fibula flap have been reported. ${ }^{4-8}$ Reasons for fibula flap reconstruction in those cases included tumors of the head or neck in four cases, tumor of the lower leg in one case, and multiple facial trauma in one case. None were cases of lower leg trauma as in the present case. Probable causes of DVT in previous reports were minor leg injury before surgery in two cases, leg immobilization after tumor biopsy in one, a long rest period after multiple injuries in one, a hypercoagulable condition associated with malignant tumor in one, and unknown cause in one. In three of six cases, the fibula flap graft was abandoned and a different reconstructive method was needed, either at the time of initial resection or at a later date. In two cases, the fibula flap was successfully grafted by using the branch to the soleus muscle as an alternative venous drainage route. In one case, the fibula flap was successfully transferred using the recanalized lumen around the chronic thrombus of the peroneal vein for anastomosis. In the present case, we removed the peroneal vein thrombi and anastomosed the vessels to the recipient posterior tibial veins; however, sufficient blood outflow was not obtained. Although the skin paddle of the flap became congested after surgery, most of the area of the skin paddle survived after exsanguination of the skin paddle with multiple skin incisions. Bone union of the grafted fibula was achieved 5 months after the surgery. In the recent large case study, the mean period for achieving bone union after the vascularized fibula graft was reported as 10 months. ${ }^{11}$ Usually, it is considered 
that achieving bone union with the nonvascularized fibula graft is difficult in the present severe trauma case. Although angiography or bone scintigraphy was not performed after surgery to demonstrate the vascularization of the grafted fibula, we considered that the fibula flap remained vascularized. This experience indicates that exsanguination is a salvage procedure when pre-existing thrombus in the peroneal vein is encountered during fibula flap harvest. Another salvage procedure to consider in this situation is inclusion of the lesser saphenous vein in the skin paddle for anastomosis as an alternative route of venous drainage.

\section{Conclusion}

A successful free osteocutaneous fibula flap might be possible in patients with peroneal venous thrombosis; however, careful preoperative evaluation of the peroneal venous system is strongly recommended for patients at high risk of developing DVT.

\section{Conflict of Interest}

None.

\section{References}

1 Taylor GI, Miller GD, Ham FJ. The free vascularized bone graft. A clinical extension of microvascular techniques. Plast Reconstr Surg 1975;55(05):533-544
2 Kessler P, Wiltfang J, Schultze-Mosgau S, Lethaus B, Greess H, Neukam FW. The role of angiography in the lower extremity using free vascularized fibular transplants for mandibular reconstruction. J Craniomaxillofac Surg 2001;29(06):332-336

3 Heit JA, Silverstein MD, Mohr DN, Petterson TM, O'Fallon WM, Melton LJ III. Risk factors for deep vein thrombosis and pulmonary embolism: a population-based case-control study. Arch Intern Med 2000;160(06):809-815

4 Taghinia AH, Pribaz JJ, Guo L. Chronic deep venous thrombosis in the peroneal veins of the fibula osteocutaneous flap: strategies for avoidance and salvage. Plast Reconstr Surg 2008;121(06):2020-2023

5 Jacobson AS, Khorsandi AS, Buchbinder D, Urken ML. Asymptomatic lower extremity deep venous thrombosis resulting in fibula free flap failure. Laryngoscope 2009;119(06):1085-1087

6 Miyamoto S, Fujiki M, Sakuraba M. Preexisting deep venous thrombosis in the peroneal vein of a free vascularized fibular graft. Plast Reconstr Surg 2013;132(04):694e-696e

7 Hsieh CH, Riva FM, Huang HY, Jeng SF. A successful free fibula transfer in the patient with chronic peroneal vein thrombosis. Ann Plast Surg 2013;71(Suppl 1):S25-S28

8 Patel SA, Abdollahi H, Ridge JA, Chang EI, Lango MN, Topham NS. Asymptomatic deep peroneal vein thrombosis during free fibula flap harvest: a review of the literature, strategies for preoperative assessment, and an algorithm for reconstruction. Ann Plast Surg 2016;76(04):468-471

9 Caprini JA. Thrombosis risk assessment as a guide to quality patient care. Dis Mon 2005;51(2-3):70-78

10 Labropoulos N, Webb KM, Kang SS, et al. Patterns and distribution of isolated calf deep vein thrombosis. J Vasc Surg 1999;30(05): 787-791

11 Houdek MT, Bayne CO, Bishop AT, Shin AY. The outcome and complications of vascularised fibular grafts. Bone Joint J 2017;99$\mathrm{B}(01): 134-138$ 